\title{
Many-anyon trial states
}

\author{
Douglas Lundholm \\ Department of Mathematics, KTH Royal Institute of Technology, SE-100 44 Stockholm, Sweden
}

(Received 30 December 2016; published 14 July 2017)

\begin{abstract}
The problem of bounding the (Abelian) many-anyon ground-state energy from above, with a dependence on the statistics parameter which matches that of currently available lower bounds, is reduced to studying the correlation functions of Moore-Read (Pfaffian) and Read-Rezayi type clustering states.
\end{abstract}

DOI: 10.1103/PhysRevA.96.012116

\section{INTRODUCTION}

The importance of the concept of quantum statistics for our understanding of observed collective phenomena in nature cannot be overstated. While fermions stack according to the Pauli principle to form a Fermi sea, with its implications for atomic structure, conduction bands in solids, etc., bosons can sit together to display amplified single-particle behavior, as manifested by Bose-Einstein condensation and the coherent propagation of light. Upon restricting to two spatial dimensions, on the other hand, it turns out to be logically conceivable to have other types of identical particles than bosons and fermions, satisfying braid statistics instead of permutation statistics, and which have been given the name "anyons" [1-5] (see also ([6], p. 386)). These have the property that under continuous exchange of two particles their wave function changes not merely by a sign \pm 1 , but any complex phase factor $e^{i \alpha \pi}$ is allowed, where the real number $\alpha$ is known as their "statistics parameter." Moreover, for logical consistency one has to keep track of any topological winding of the particles during their exchange. For instance, if one particle moves around in configuration space in such a way as to enclose $p$ other particles in a complete (counterclockwise, say) loop, a phase $2 p \alpha \pi$ must arise, while if two particles are exchanged once and in the process $p$ other particles are enclosed, the phase must be $(1+2 p) \alpha \pi$. All such topological complications vanish in the case of $\alpha=0$ (bosons) and $\alpha=1$ (fermions). The concept has also been extended from phases (Abelian) to unitary matrices (non-Abelian), but we shall here stick to the simpler (though not at all simple) Abelian case. Furthermore, instead of demanding that the wave function changes its phase according to the above form of topological boundary conditions, also known as the "anyon gauge picture," one may equivalently model such phases by means of attaching magnetic flux to ordinary identical particles, i.e., bosons or fermions, resulting in a magnetic many-body interaction. This is then called the "magnetic gauge picture" for anyons. We refer to the extensive reviews [7-16] for a more complete background on the topic.

The idea of particles with attached magnetic flux is fundamental to the fractional quantum Hall effect (FQHE)

Published by the American Physical Society under the terms of the Creative Commons Attribution 4.0 International license. Further distribution of this work must maintain attribution to the author(s) and the published article's title, journal citation, and DOI.
[17-21], which is a manifestation of a strongly correlated many-body state of electrons subject to planar confinement and a strong transverse magnetic field. More recently it has been proposed that similar effects apply to trapped bosonic atoms in artificial magnetic fields [22-26], and to graphene $[27,28]$. The quasiparticles arising in the FQHE of electrons are predicted to be anyons with a corresponding fractional statistics parameter. However, as discussed in ([19], Sec. 9.8.2), there has been some confusion in the literature concerning the exact values of $\alpha$ involved. This can be traced to different conventions but also to the fact that the statistics parameter has hitherto only been defined indirectly via the operation of adiabatic braiding and the computation of a corresponding Berry phase, as first outlined in [29]. Only very recently has an effective Hamiltonian for anyons been derived [30], which shows unambiguously how they may arise in a FQHE context and what the statistics parameter then should be, confirming that fermions against the background of a fermionic Laughlin state with odd exponent $2 p+1$ effectively couple to Laughlin quasiholes to behave as emergent anyons with $\alpha=2 p /(2 p+1)$ (see also [31]).

Despite the concept of anyons having been around now for almost four decades, a satisfactory understanding of their physics is still lacking. Due to their complicated many-body interaction (or geometry) it has not been possible to solve the anyon Hamiltonian for its complete spectrum or even its ground state, except in the two-particle case where it can be reduced to a one-particle problem and thus be solved analytically $[1,4,32]$, while in the three- and four-particle cases it has been studied numerically [33-36]. Nevertheless, as is evident from the vast body of literature (the author can count more than 300 papers on the topic), there has been a fair amount of progress on the many-anyon problem, most of which is based on various approximations. One of the most discussed is average-field theory (see, e.g., Refs. [10,16,37-39] for review), where the individual anyons are replaced by their average magnetic field, something which is arguably reasonable in a sufficiently dense regime. Other approximations assume either a very strong external magnetic field, thereby reducing to lowest-Landau-level anyons which turn out to be solvable with a connection to Calogero-Sutherland models [14,40], or in the case of the free dilute gas, that it is sufficient to only take two-particle interactions into account $[32,41]$. It has however been stressed that real progress in understanding the anyon gas cannot be made without knowledge of the true many-body spectrum.

In a recent series of works [42-47], the question concerning the many-anyon ground state has been investigated in the 
light of modern mathematical methods. Interestingly, it was found that the ground-state energy for the free ideal anyon gas can be nontrivially bounded from below, but only under the assumption that $\alpha$ is an odd-numerator rational number (in contrast to electron FQHE which typically involves odddenominator filling factors). To settle the issue whether this is the true behavior or rather just an artifact of the method used to obtain the bounds, one also needs to bound the energy from above using suitable trial states. This however turns out to be a very difficult problem for anyons, contrary to the more common situation where finding the upper bound is the easier part.

We shall here proceed in the setting of Abelian anyons with no external magnetic field (which is indeed relevant in the FQHE context; cf., e.g., Ref. [15]), and propose, building on [43], that good variational ground states for the many-anyon problem are given by clustering states of the Moore-Read and Read-Rezayi type that have already been studied for some time in the context of special (proposedly non-Abelian) regimes of the FQHE. In particular, these types of states seem to give a much lower energy for even-numerator rational $\alpha$ than for odd numerators, and offer a corresponding picture of condensation, respectively, a reduced Fermi sea of anyons. In view of the above considerations, such a picture could in the context of the FQHE potentially have far-reaching consequences.

\section{MANY-ANYON GROUND-STATE ENERGY}

For concreteness and for easier comparison with the results which are available in the literature, we consider as our starting point anyons confined in a harmonic-oscillator potential. In the magnetic gauge, the Hamiltonian operator for $N$ nonrelativistic ideal ${ }^{1}$ anyons with mass $m$ in a harmonic trap with frequency $\omega \geqslant 0$, and in units such that $\hbar=1$, is

$$
\hat{H}_{N}=\hat{T}_{\alpha}+\hat{V}=\sum_{j=1}^{N}\left(\frac{1}{2 m} D_{j}^{2}+\frac{m \omega^{2}}{2}\left|\mathbf{x}_{j}\right|^{2}\right)
$$

where

$$
D_{j}:=-i \nabla_{\mathbf{x}_{j}}+\alpha \mathbf{A}_{j}\left(\mathbf{x}_{j}\right)
$$

denotes the magnetically coupled momentum operator of particle $j$ at position $\mathbf{x}_{j} \in \mathbb{R}^{2}$. Each particle sees an AharonovBohm magnetic flux $2 \pi \alpha$ attached to every other particle, as is given explicitly by the magnetic potentials

$$
\mathbf{A}_{j}(\mathbf{x}):=\sum_{\substack{k=1 \\ k \neq j}}^{N}\left(\mathbf{x}-\mathbf{x}_{k}\right)^{-\perp}, \quad \mathbf{x}^{-\perp}:=\frac{\mathbf{x}^{\perp}}{|\mathbf{x}|^{2}}=\frac{(-y, x)}{x^{2}+y^{2}} .
$$

For reference we take the Hamiltonian $\hat{H}_{N}$ to act on bosonic states $\Psi \in L_{\text {sym }}^{2}\left(\left(\mathbb{R}^{2}\right)^{N}\right)$, and there is associated with the free kinetic energy operator $\hat{T}_{\alpha}$ a natural subspace (form domain) $\mathscr{D}_{\alpha}^{N}$ consisting of the states $\Psi$ which have a finite expectation value for their kinetic energy (see [44], Sec. 2.2 and [46], Sec. 1.1 for details). The case $\alpha=0$ then corresponds to

\footnotetext{
${ }^{1}$ That is, without interactions other than the statistical one, and purely pointlike as opposed to extended; cf. Sec. VI below.
}

bosons and $\alpha=1$ to fermions, with $\mathscr{D}_{\alpha=0}^{N}=H_{\text {sym }}^{1}\left(\mathbb{R}^{2 N}\right)$ and $\mathscr{D}_{\alpha=1}^{N}=U^{-1} H_{\text {asym }}^{1}\left(\mathbb{R}^{2 N}\right)$ the Sobolev spaces of symmetric respectively antisymmetric square-integrable functions having square-integrable first derivatives. In the latter case we have used the singular gauge transformation $U^{-1} \nabla_{\mathbf{x}_{j}} U=i \mathbf{A}_{j}$, with

$$
\begin{aligned}
U: L_{\text {sym/asym }}^{2} & \rightarrow L_{\text {asym/sym }}^{2}, \\
(U \Psi)(x) & :=\prod_{1 \leqslant j<k \leqslant N} \frac{z_{j}-z_{k}}{\left|z_{j}-z_{k}\right|} \Psi(x),
\end{aligned}
$$

and with the coordinates here represented by $z_{j}=x_{j}+$ $i y_{j} \in \mathbb{C}$, to explicitly switch from fermions to their bosonic representation via flux attachment. The same transformation can be used to show that the full spectrum must be periodic in $\alpha$ up to any even integer $2 q$, by composing $\Psi$ with $U^{-2 q}$ which preserves symmetry. Also, one could equivalently have chosen to model everything in terms of fermions with statistics parameter $\beta:=\alpha-1$.

As is very well known, the harmonic-oscillator ground-state energy

$$
E_{0}(N):=\inf \operatorname{spec} \hat{H}_{N}=\inf _{\Psi \in \mathscr{D}_{\alpha}^{N} \backslash\{0\}} \frac{\left\langle\Psi, \hat{H}_{N} \Psi\right\rangle}{\langle\Psi, \Psi\rangle}
$$

is for bosons $E_{0}(N)=\omega N$, while for (spinless) fermions $E_{0}(N) \sim \frac{\sqrt{8}}{3} \omega N^{3 / 2}$ as $N \rightarrow \infty$ due to the Pauli principle and the filling of one-body states. For fermions allowed to have $v \geqslant 1$ different spin states (or particles obeying Gentile intermediate statistics $[48,49])$ it is a simple exercise to show using the same asymptotics that $E_{0}(N) \sim \frac{\sqrt{8}}{3} v^{-1 / 2} \omega N^{3 / 2}$. However, for anyons with statistics parameter $\alpha$ it has now been established $[42-44,46]$ that

$$
C_{1} j_{\alpha_{N}}^{\prime} \omega N^{3 / 2} \leqslant E_{0}(N) \leqslant C_{2} \omega N^{3 / 2}
$$

for some universal constants $C_{1} \leqslant \sqrt{8} /\left(3 j_{1}^{\prime}\right)$ and $C_{2} \geqslant \sqrt{8} / 3$. Here $j_{a}^{\prime}$ for $a>0$ denotes the first positive zero of the derivative of the Bessel function $J_{a}$ of the first kind, satisfying (see [46])

$$
\sqrt{2 a} \leqslant j_{a}^{\prime} \leqslant \sqrt{2 a(1+a)} \quad\left(\text { and } j_{0}^{\prime}:=0\right) .
$$

The order $a=\alpha_{N}$ involved is given by the "fractionality" of $\alpha$ as measured by

$$
\alpha_{N}:=\min _{p \in\{0,1, \ldots, N-2\}} \min _{q \in \mathbb{Z}}|(2 p+1) \alpha-2 q| .
$$

This expression has the peculiar many-body limit [42, Prop. 5] (see Fig. 1)

$$
\begin{aligned}
\alpha_{*} & :=\lim _{N \rightarrow \infty} \alpha_{N}=\inf _{N \geqslant 2} \alpha_{N} \\
& = \begin{cases}\frac{1}{v}, & \text { if } \alpha=\frac{\mu}{v} \in \mathbb{Q} \text { reduced, } \mu \text { odd and } v \geqslant 1, \\
0, & \text { otherwise. }\end{cases}
\end{aligned}
$$

In particular, the factor in front of $\omega N^{3 / 2}$ in the lower bound in (3) depends on $\alpha$ as $\sqrt{\alpha_{*}}=v^{-1 / 2}$ for small odd-numerator fractions and tends to zero with $N$ for even-numerator and irrational numbers.

In addition to the above, for an arbitrary state $\Psi$ with fixed total angular momentum $L \in \mathbb{Z}$ one also has the bound 

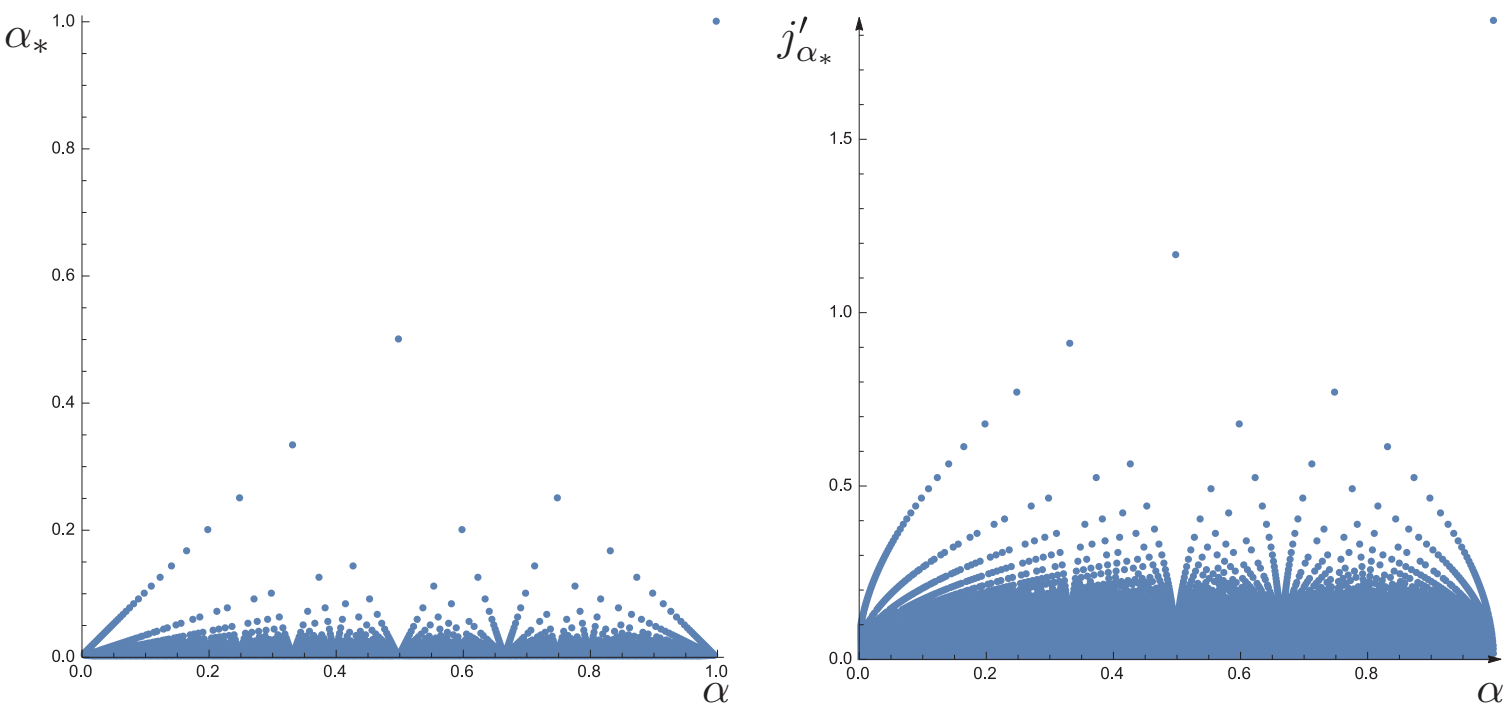

FIG. 1. Plots of $\alpha_{*}$ respectively $j_{\alpha_{*}}^{\prime}$ for $0 \leqslant \alpha \leqslant 1$. These can be continued to all $\alpha \in \mathbb{R}$ using periodicity and reflection (conjugation) symmetry.

\section{$[13,50,51]$}

$$
\left\langle\Psi, \hat{H}_{N} \Psi\right\rangle \geqslant \omega\left(N+\left|L+\alpha \frac{N(N-1)}{2}\right|\right),
$$

where, if $L=-\alpha\left(\begin{array}{c}N \\ 2\end{array}\right)$ (i.e., an average angular momentum of $-\alpha$ per particle pair) would be achieved exactly on some state, then the remaining bound is just the ground-state energy for bosons. As discussed in [50], since $E_{0}(N)=O\left(N^{3 / 2}\right)$ the bound (5) implies that as the number of particles increases there will be more and more level crossings in the ground state to different angular momenta, resulting in a qualitative picture (see [50, Fig. 1]) with some interesting features in common with Fig. 1 . Note that, for every finite $N$, the ground-state curve is conjectured to be continuous in $\alpha$ and piecewise smooth; however, the number of such pieces grows like $\sqrt{N}$.

On the other hand, an approach that has been used extensively in the literature to understand the anyon gas (see the reviews and references therein) is to employ the "average-field approximation" 2

$$
\begin{aligned}
E_{0}(N) & \approx \inf _{\substack{\varrho \geqslant 0 \\
\int \varrho=N}} \int_{\mathbb{R}^{2}}\left(\frac{\pi|\alpha|}{m} \varrho(\mathbf{x})^{2}+\frac{m \omega^{2}}{2}|\mathbf{x}|^{2} \varrho(\mathbf{x})\right) d \mathbf{x} \\
& =\frac{\sqrt{8}}{3} \sqrt{|\alpha|} \omega N^{3 / 2} .
\end{aligned}
$$

Here it has been assumed that the anyons see each other via an approximately constant magnetic field $B(\mathbf{x}) \sim 2 \pi \alpha \varrho(\mathbf{x})$, with $\varrho(\mathbf{x})$ the local density of particles, and hence they each have a lowest-Landau-level energy $|B| /(2 m) \sim \pi|\alpha| \varrho / m$. In [45] it has been shown rigorously that such an approximation is indeed correct in the limit of "almost-bosonic" anyons (i.e., $\alpha \rightarrow 0$; see also [47]) in a confining trap; however,

\footnotetext{
${ }^{2}$ This is also known as the mean-field approximation in the literature, although it is useful to make a distinction between the names; cf. [16,45].
}

one needs to be careful with what is meant exactly with the approximation and how such a limit is performed since strictly speaking the anyons cannot be ideal but extended (see [52] and Sec. VI below). Also note that the periodicity for ideal anyons mentioned above is not naturally implemented in (6), so we must at least expect to replace $\alpha$ with its periodization $\alpha_{2}$ from (4).

The main question raised from the bounds (3) and (5) is whether for certain $\alpha$ such that $\alpha_{*} \ll \alpha_{2}$-most notably for even-numerator rational $\alpha$ such as $\alpha=2 / 3$, for which $\alpha_{*}=$ 0 - and for particular states such that $L \sim-\alpha\left(\begin{array}{c}N \\ 2\end{array}\right)$, the true ground-state energy $E_{0}(N)$ could be considerably lower than the one (6) expected from average-field theory. Building on [43], we shall here explore the possibility that this is actually the case.

\section{LOCAL EXCLUSION PRINCIPLE FOR ANYONS}

In order to understand the origin of the peculiar dependence of the above energy bounds on $\alpha_{*}$, and of the form of the corresponding proposed trial states, we first need to briefly discuss the findings in $[42,43,46]$ of a local exclusion principle for anyons.

Normally when one talks about an exclusion principle for identical particles one has in mind an occupation picture, where only a limited number of particles can sit in each distinguishable one-body state. The prime example is of course the usual Pauli exclusion principle for fermions, although various extensions have also been discussed in the literature $[40,48,49,53-56]$ (the role of such generalized exclusion in the context of anyons has also been reviewed in [57]). Sometimes the notion is generously extended to concern exclusion of coincident points in the configuration space, and anyons have often been pointed out to obey such exclusion, either because it is required for their topological definition or because their singular (for ideal anyons) magnetic interaction forces the wave function to vanish on the diagonals, just like fermions do by means of antisymmetry (see also [58]). However, note that such a notion of exclusion also applies to the hard-core 
Bose gas whose thermodynamic ground-state energy vanishes in the dilute limit (in analogy with the noninteracting gas) in two and higher dimensions [59-64].

For anyons whose statistics is generated by a true manybody interaction (or a very complicated geometry), a stronger notion of exclusion is required, and such a notion has been developed in $[42,43,46]$ in the form of an effective repulsive long-range pair interaction. Namely, recall that the effect of the statistics is a change of phase of the anyonic wave function by $e^{i \alpha \pi}$ whenever two particles are interchanged via a simple continuous loop in configuration space, or a total phase $(1+2 p) \alpha \pi$ whenever such an exchange loop at the same time encloses $p$ other particles. On the other hand, particles are also allowed to have pairwise relative angular momenta, and such momenta are by continuity restricted to only change the phase by an even multiple of $\pi$. One way of viewing this condition (in the magnetic gauge picture) is that the many-body wave function is modeled as a bosonic (or fermionic) one and thus it needs to be antipodal-symmetric (antisymmetric) with respect to the relative coordinate, hence $\pi$-periodic (antiperiodic) in the relative angle. Another way to see it (in the anyon gauge or geometric picture) is that the wave function is a section of a locally flat complex line bundle with the topological continuity condition that its phase around such a loop should jump by $(1+2 p) \alpha \pi$ plus $2 \pi$ times an arbitrary winding number. Assuming then that the relative momentum or winding is the even integer $-2 q$ if the particles are orbiting in a reversed (for $\alpha>0$ ) direction in order to cancel as much of the magnetic or topological phase as possible, we arrive at a total phase $(2 p+1) \alpha-2 q$ times $\pi$ per exchange for the particle pair. In the kinetic energy, any nonzero remainder phase of this sort gives rise to a centrifugal-barrier repulsion

$$
V_{\text {stat }}(r)=|(2 p+1) \alpha-2 q|^{2} \frac{1}{r^{2}} \geqslant \frac{\alpha_{N}^{2}}{r^{2}} \geqslant \frac{\alpha_{*}^{2}}{r^{2}},
$$

where $r$ denotes the relative distance of the particle pair, and we have taken the infimum over all possibilities $p \in$ $\{0,1, \ldots, N-2\}$ and $q \in \mathbb{Z}$ to obtain the lower bounds in terms of $\alpha_{N} \geqslant \alpha_{*}$. In the case of $\alpha=\mu / \nu$ being an arbitrary reduced fraction with an odd numerator $\mu$ and a positive denominator $v$, it turns out using simple number theory ([42] Prop. 5) that this phase mismatch can never be completely canceled, and in fact $\alpha_{*}=1 / \nu>0$. On the other hand, if $\mu$ is an even number it is evident that cancellation is possible for particular values of $p$ and $q$, and therefore $\alpha_{*}=0$. For irrational values of $\alpha$, one can use that any such number can be approximated arbitrarily well by both even- and odd-numerator rational numbers and hence $\alpha_{*}=0$ (although note that a very large $N$ may be required in such a process).

A geometric interpretation of the potential (7) is that there is nontrivial curvature (magnetic flux) sitting at each of the enclosed particles but effectively seen at the center $r=0$ of the particle pair, and its presence is felt by the kinetic energy in the form of an effective potential. The situation is from this perspective analogous to that of a free quantum particle moving on a cone [65-67], here with its apex angle depending on $\alpha$ and the number of enclosed particles.

In [42] and [46] it has been shown rigorously by means of a family of magnetic Hardy inequalities that such an effective pairwise inverse-square "statistical repulsion" (7) indeed arises

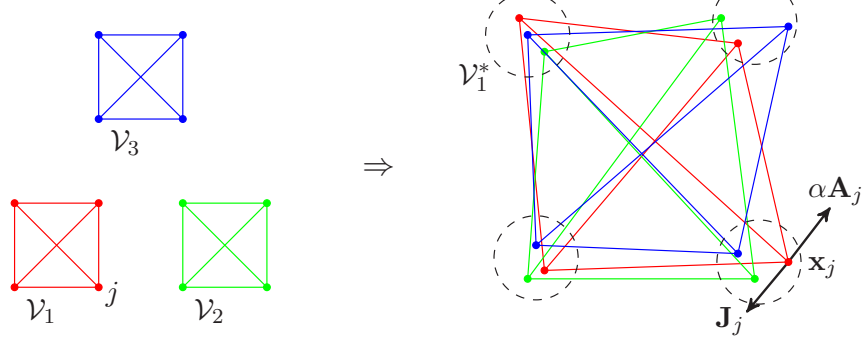

FIG. 2. Illustration of a coloring of $N=12$ particles with $v=3$ colors into $K=4$ clusters. Each colored edge corresponds to one unit $-\mu$ of pairwise angular momentum. Also shown is the contribution to the magnetic potential $\alpha \mathbf{A}_{j}$ and the current $\mathbf{J}_{j}$ of particle $j$ due solely to the cluster $\mathcal{V}_{1}^{*}$.

in the many-anyon system. Although the effect is in some sense local and weighted only linearly in the number of particles (in contrast to a usual pair-interaction term in the Hamiltonian), it is still of long-range type and sufficiently strong to produce a "degeneracy pressure" (represented concretely in the form of Lieb-Thirring inequalities; cf. [43,68]) and consequently nontrivial energy bounds in terms of $\alpha_{*}$ for the ideal or dilute anyon gas, such as (3). We also stress that the method used to obtain the effective pair potential (7), which was introduced in [42] and developed to encompass more general situations in [46], is well suited for numerical investigations of lower bounds to the ground-state energy.

\section{CONSTRUCTING ANYONIC TRIAL STATES}

In order to match the available lower bounds for $E_{0}(N)$ from above, it was in [43] suggested to study variational trial states of the form

$$
\Psi=\Phi \psi_{\alpha} \in L_{\mathrm{sym}}^{2}\left(\mathbb{R}^{2 N}\right),
$$

with $\Phi \in L_{\text {loc,sym }}^{1}$ a locally integrable regularizing factor, $N=$ $\nu K$ a suitable sequence of particle numbers, and, in the case of $\alpha$ being an even-numerator reduced fraction $\alpha=\mu / \nu \in[0,1]$,

$$
\psi_{\alpha}:=\prod_{j<k}\left|z_{j k}\right|^{-\alpha} \mathcal{S}\left[\prod_{q=1}^{v} \prod_{(j, k) \in \mathcal{E}_{q}}\left(\bar{z}_{j k}\right)^{\mu}\right] \prod_{l=1}^{N} \varphi_{0}\left(\mathbf{x}_{l}\right),
$$

while for $o d d$ numerators $\mu$,

$$
\psi_{\alpha}:=\prod_{j<k}\left|z_{j k}\right|^{-\alpha} \mathcal{S}\left[\prod_{q=1}^{v} \prod_{(j, k) \in \mathcal{E}_{q}}\left(\bar{z}_{j k}\right)^{\mu} \bigwedge_{k=0}^{K-1} \varphi_{k}\left(\mathbf{x}_{l \in \mathcal{V}_{q}}\right)\right] .
$$

Here $z_{j k}:=z_{j}-z_{k}$ are the pairwise relative complex coordinates with the usual identification $\mathbb{C} \ni z_{j} \leftrightarrow \mathbf{x}_{j} \in \mathbb{R}^{2}$, and we have grouped, or "colored," the particles into $v$ different colors where $G_{q}=\left(\mathcal{V}_{q}, \mathcal{E}_{q}\right)$ denotes the complete graph over each such group of $\left|\mathcal{V}_{q}\right|=K$ vertices=particles (cf. Fig. 2). The symmetrization $\mathcal{S}$ over all the particles then amounts to symmetrization over all such colorings, and can be viewed as passing from a set of distinguishable particles (by color) to indistinguishable (cf. [69]). The $\varphi_{k}, k=0,1,2, \ldots$, are the eigenstates (ordered by increasing energy) of a corresponding 
one-body Hamiltonian

$$
\hat{H}_{1}=\frac{1}{2 m}\left(-i \nabla_{\mathbf{x}}+\mathbf{A}_{\mathrm{ext}}(\mathbf{x})\right)^{2}+V(\mathbf{x}),
$$

and in (9) we have formed the Slater determinant of the $K$ first such states in the variables of each color group $\mathcal{V}_{q}$ to obtain matching symmetry.

In the harmonic-oscillator case $\mathbf{A}_{\text {ext }}=0, \quad V(\mathbf{x})=$ $m \omega^{2}|\mathbf{x}|^{2} / 2$, these $N$-body states have angular momentum

$$
L=-\mu \nu\left(\begin{array}{l}
K \\
2
\end{array}\right)=-\alpha\left(\begin{array}{l}
N \\
2
\end{array}\right)+\alpha \frac{v-1}{2} N,
$$

for (8) and for certain "magic" numbers $K$ in (9) corresponding to filled shells. The symmetrized quantity in (8) is then a homogeneous polynomial in $\bar{z}_{j}$ which, multiplied with the Gaussian $e^{-m \omega|z|^{2} / 2},|z|^{2}=\sum_{j=1}^{N}\left|z_{j}\right|^{2}$, coincides exactly with the (complex-conjugated bosonic) LaughlinRead-Rezayi states for the fractional quantum Hall effect [69-71]. Also note that, if the $\varphi_{k}$ are instead taken to be the lowest-Landau-level states of a constant magnetic field with cyclotron frequency $\omega$ in symmetric gauge,

$$
\varphi_{k}(z) \propto \bar{z}^{k} e^{-m \omega|z|^{2} / 2}, \quad k=0,1,2, \ldots,
$$

then the case $\alpha=1 / 2$ in (9) corresponds to the Moore-Read (Pfaffian) states [71,72] (cf. also [39,73] where pairing of semions has been discussed).

It is known that these states possess clustering properties, so that for example the symmetric polynomial ${ }^{3}$

$f_{N=\nu K}(\mathrm{z}):=\frac{1}{(\nu !)^{K-1}} \mathcal{S}\left[\prod_{q=1}^{\nu} \prod_{(j, k) \in \mathcal{E}_{q}}\left(z_{j k}\right)^{\mu}\right], \quad \mu=2,4,6, \ldots$

(together with a confining factor such as the Gaussian) exhibits clusters of $v$ particles. This, as well as other interesting and useful properties of such symmetric polynomials, have also been observed by means of an identification with correlators of certain conformal field theories (see, e.g., Refs. [71,74,75]) and with Jack polynomials [76]. For instance, one has that if the positions of $v$ particles (i.e., one cluster) are identified, then (also compare to Fig. 2)

$$
\begin{aligned}
& f_{N}(\underbrace{\zeta, \ldots, \zeta}_{\nu \text { copies }}, z_{v+1}, z_{v+2}, \ldots, z_{N}) \\
& \quad=\prod_{j=v+1}^{N}\left(\zeta-z_{j}\right)^{\mu} f_{N-v}\left(z_{\nu+1}, \ldots, z_{N}\right) .
\end{aligned}
$$

In particular, $f_{N}$ then vanishes whenever $v+1$ or more particles are brought together. Furthermore, if proceeding in this way to group all particles into disjoint clusters $\mathcal{V}_{q}^{*}$, $q=1, \ldots, K$, with $\left|\mathcal{V}_{q}^{*}\right|=v$ [think of complete graphs $G_{q}^{*}=$ $\left(\mathcal{V}_{q}^{*}, \mathcal{E}_{q}^{*}\right)$ dual to $G_{q}$ in a sense], and then identifying their positions, say $z_{j}=\zeta_{q}$ for $j \in \mathcal{V}_{q}^{*}$, one obtains a Laughlin state

\footnotetext{
${ }^{3}$ The normalization factor here is chosen to simplify the identities below. The number of terms in the symmetrized expression is $(v K) ! /\left[v !(K !)^{v}\right]$.
}

with exponent $v^{2} \alpha$,

$$
f_{N}(z)=\prod_{1 \leqslant p<q \leqslant K}\left(\zeta_{p}-\zeta_{q}\right)^{v \mu} .
$$

Note that this clustering behavior matches very well with both the form of the magnetic potential $\mathbf{A}_{j}$ and the Jastrow prefactor in $\psi_{\alpha}$. Namely, the attractive Jastrow factor contracts the clusters and balances with the intercluster repulsion coming from the Jack polynomial $f_{N}(\bar{z})$ in such a way that each particle $\mathbf{x}_{j}$ sees from any other cluster $\mathcal{V}_{q}^{*} \notin j$, say located at $\mathbf{y} \leftrightarrow \zeta$ at a large distance $r=|\mathbf{r}|=\left|z_{j}-\zeta\right|$, the attraction $\prod_{k \in \mathcal{V}_{q}^{*}}\left|z_{j k}\right|^{-\alpha} \sim r^{-v \alpha}=r^{-\mu}$ and at the same time the effective repulsion $\sim\left|z_{j}-\zeta\right|^{\mu}=r^{\mu}$ from (12). Also, the total contribution to the magnetic potential seen by particle $\mathbf{x}_{j}$ from this cluster is $\alpha \mathbf{A}_{j}\left(\mathbf{x}_{j} ; \mathbf{x}_{k \in \mathcal{V}_{q}^{*}}\right) \sim v \alpha \mathbf{r}^{-\perp}$, while at the same time the particle has an orbital angular momentum around the cluster with an opposite current contribution $\mathbf{J}_{j}=-i \psi_{\alpha}^{-1} \nabla_{j} \psi_{\alpha}\left(\mathbf{x}_{j} ; \mathbf{x}_{k \in \mathcal{V}_{q}^{*}}\right) \sim-\mu \mathbf{r}^{-\perp}$, again thanks to (12) (and complex conjugation). One should also observe that (see Fig. 2), due to the balance between Jastrow attraction and Jack repulsion, clusters are formed out of particles with different colors, i.e., in different groups $\mathcal{V}_{q}$. Furthermore, every particle has exactly one edge in $\mathcal{E}_{q}$ going to exactly one particle in every other cluster, namely the particle of the same color, and this is what gives the orbital angular momentum contribution $\left(\bar{z}_{j k}\right)^{\mu}$. In this way there is a natural cancellation between magnetic flux and angular momentum on the level of each individual particle. The same holds in the case of the odd-numerator states, which however have an additional repulsion and possibly angular momentum coming from the Slater determinant in (9).

In the fully clustered picture (13),

$$
\Psi=\Phi \psi_{\alpha} \sim \prod_{1 \leqslant p<q \leqslant K}\left(\frac{\overline{\zeta_{p}-\zeta_{q}}}{\left|\zeta_{p}-\zeta_{q}\right|}\right)^{v \mu}
$$

becomes the necessary gauge transformation $U^{-v \mu}$ to remove the overall statistical effects of the clusters (here we have $v$ copies of the even integer $\mu$ because there are $\nu$ particles moving in each cluster, each seeing a flux $\nu \alpha=\mu$ from every other cluster). Note that the role of $\Phi$ is to regularize the singular short-scale dependence of $\psi_{\alpha}$ arising upon bringing particles very close together (the Jastrow factor in $\psi_{\alpha}$ diverges with each pair like $\left|z_{j k}\right|^{-\alpha}$ ), and in (14) we have assumed that this has effectively removed the singular factor $\prod_{(j, k) \in \mathcal{E}_{q}^{*}}\left|z_{j k}\right|^{-\alpha}$ from within each cluster. We also note that the states (8) and (9) naturally generalize for $\alpha \in \mathbb{Z}$ to the correct gauge copies $\Psi=U^{-\alpha} \Psi_{0}$ of the bosonic (fermionic) ground states, $\Psi_{0}=\otimes^{N} \varphi_{0}\left(\Psi_{0}=\wedge_{k=0}^{N-1} \varphi_{k}\right)$, in this case leaving out the need for the regulator $\Phi$.

With the assumption that the total energy increases with the total degree of one-particle states $\varphi_{k}$, the odd-numerator states clearly have a higher energy than the even-numerator ones, simply enforced by the symmetry constraint. One needs to explain, however, why one cannot just take the same states $\psi_{\alpha}$ but shifted to a reducible fraction $\alpha=\mu / \nu=\mu^{\prime} / \nu^{\prime}$, with $\mu^{\prime}=$ $k \mu, v^{\prime}=k \nu$, and $k \geqslant 2$. Note first that the necessary properties of the states may not be valid for such fractions and indeed there are certain assumptions on irreducibility in the context 

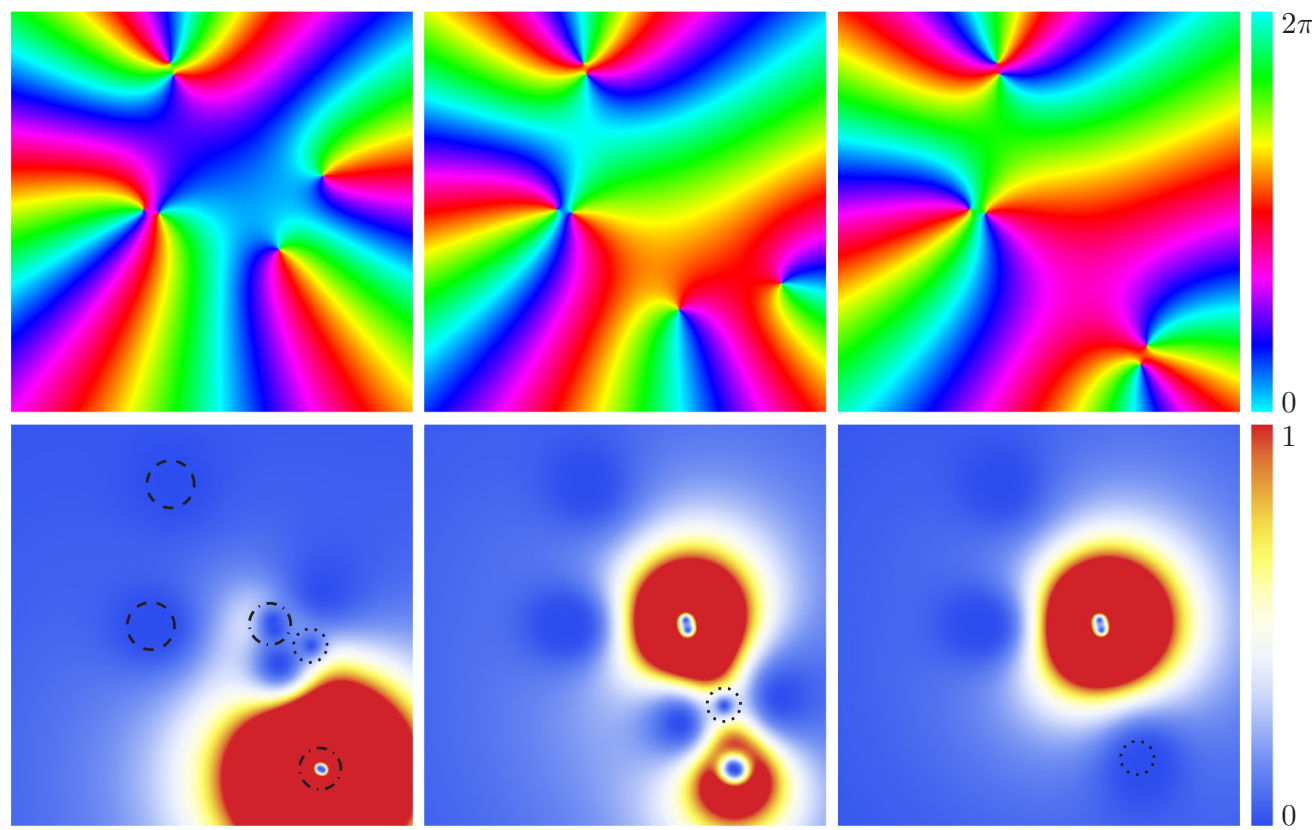

FIG. 3. Sequence of three particle configurations chosen to illustrate the properties of the trial states $\Psi$, here with $\alpha=2 / 3, N=12$, and $\Phi=\Phi_{r_{0}=1.3}$ as defined in (17). We fix the positions of 11 particles in two three-clusters (dashed circles), two two-clusters (dash-dotted circles), and a single particle at three intermediate positions between the two-clusters (dotted circles). Then $\arg \Psi$ (top row) and $|\Psi|^{2}$ (bottom row; normalization chosen for illustration) are plotted as functions of the remaining particle position on the square $[-10,10]^{2}$. One may note that each three-cluster binds two vortices, which produce the desired orbital angular momentum and also cancel the attraction from the cluster very effectively. The attraction is much stronger from a two-cluster than from a single particle, but entirely absent from three-clusters.

of Jack polynomials [76], but let us proceed anyway with the discussion, aiming for a better understanding of the argument.

Within the class of even or odd states we expect that the size of clusters, i.e., the denominator $v$ respectively $v^{\prime}$, dictates the energy of the regulator $\Phi$ which therefore favors the irreducible case $k=1$. Also note that we cannot shift from an even state to an odd one by extending the fraction by $k$ in this way, but one could certainly take $k$ to be even and thereby shift an odd state into an even one, and thus argue that the energy should then become lower (consider, for example, $\alpha=2 / 6$ instead of $1 / 3$ or, for $\alpha=1$, Cooper pairs instead of a single Slater determinant of fermions). To argue against this possibility we need to study the pairwise structure of the states closer.

First note that the pairwise relative angular momentum for any (shifted or not) even state $\psi_{\alpha=\mu^{\prime} / v^{\prime}}$ always comes in multiples of $\mu^{\prime}$. The statistical repulsion (7) then always gives a positive energy unless for some $p, q \in\{0,1,2, \ldots\}$

$$
(1+2 p) \alpha=q \mu^{\prime} \Rightarrow q v^{\prime}=1+2 p,
$$

which requires $v^{\prime}$ to be odd, so that $k=1$ (and $v$ is already odd if $\mu$ was even). We also note that if any $\nu$-particle clusters happen to be enclosed in the two-anyon exchange loops, they each contribute $v$ to $p$, that is $2 v \alpha$ to the magnetic phase, and at the same time a matching $-2 \mu$ to the relative momentum (one $-\mu$ for each of the anyons orbiting around the cluster). Furthermore, on a length scale such that a typical pair of particles has exactly one multiple of $\mu$ as orbital angular momentum, a full cancellation with the magnetic phase demands $p=(v-1) / 2$ (this is indeed an integer in the even-numerator case), i.e., about half of the particles of a cluster are enclosed. On the very smallest scales, i.e., much less than the average interparticle distance, we can accept a phase mismatch and strong repulsion, to be controlled by $\Phi$, in analogy with, e.g., the hard-core Bose gas whose energy vanishes logarithmically with low density in two dimensions $[61,63]$. In the case $\alpha=2 / 3$ it is natural that as the scale then increases a bit we first see exactly one enclosed anyon, $p=1$, of the $v=3$ cluster of which the particle pair is taken, and then additional full clusters on the scale of the average interparticle distance (cf. [46], Fig. 3 and Fig. 4). Hence this allows for a full cancellation of the exchange phase over large scales in this particular state, thus reducing its overall statistical repulsion on large regions of the configuration space. However, in general it seems that there needs to be a delicate balance between $\Phi$ and $\psi_{\alpha}$ in order to obtain such special probability distributions, and this remains the least understood aspect of these trial states at the moment (see also [77]).

\section{IDEAL ANYONS IN A HARMONIC TRAP}

At this point one might worry about actually computing (or at least bounding) the energy of the proposed trial states. Fortunately, however, it turns out that $\psi_{\alpha}$ given in (8) for even-numerator $\alpha$ is an exact (but singular) eigenfunction of the harmonic-oscillator Hamiltonian $\hat{H}_{N}$ with (see [13,78-80])

$$
\hat{H}_{N} \psi_{\alpha}=\omega\left(N+\operatorname{deg} \psi_{\alpha}\right) \psi_{\alpha},
$$

where the degree of the state is, by (11),

$$
\operatorname{deg} \psi_{\alpha}=-\alpha\left(\begin{array}{l}
N \\
2
\end{array}\right)-L=-\alpha \frac{v-1}{2} N .
$$



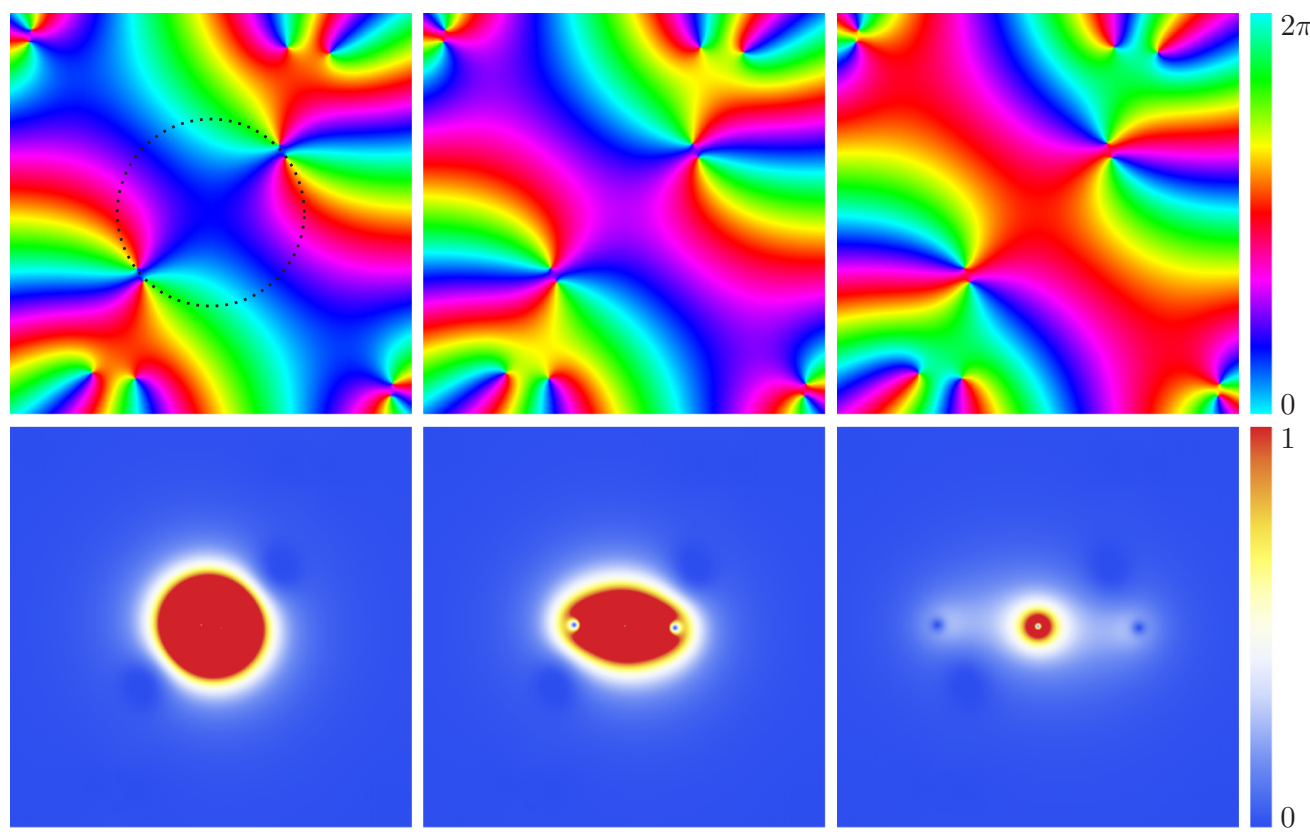

FIG. 4. Similar plot as in Fig, 3 with the same state $\Psi$. Now three three-clusters are fixed, one particle takes three different positions along the horizontal axis, while the relative coordinate of the remaining pair of particles is plotted on the square $[-20,20]^{2}$ with the center of mass of the pair fixed at the origin. In relative coordinates the picture is antipodal symmetrized, and one may also note that on a circle with radius equal to the nearest three-cluster distance (dotted) the total phase circulation is $4 \pi$. In this way the probability of finding a third particle within an interparticle distance may possibly be increased without a significant cost in exclusion energy.

Being a singular eigenfunction means that the identity (15) holds wherever $\psi_{\alpha}$ is smooth, namely outside of the fat diagonal of the configuration space

$$
\begin{aligned}
\Delta & :=\left\{\mathrm{x}=\left(\mathbf{x}_{1}, \ldots, \mathbf{x}_{N}\right)\right. \\
& \left.\in\left(\mathbb{R}^{2}\right)^{N}: \exists j \neq k \text { such that } \mathbf{x}_{j}=\mathbf{x}_{k}\right\} .
\end{aligned}
$$

Since $\psi_{\alpha}$ is not a true eigenstate (normalizable and in the domain) of the operator $\hat{H}_{N}$, its formal energy being

$$
\omega\left(N+\operatorname{deg} \psi_{\alpha}\right)=\omega\left(1-\alpha \frac{v-1}{2}\right) N<\omega N
$$

is not a contradiction to (5). Also, by contrast, note that for $\psi_{\alpha}$ with odd-numerator $\alpha$

$$
\operatorname{deg} \psi_{\alpha} \sim v \frac{\sqrt{8}}{3} K^{3 / 2}=O\left(N^{3 / 2}\right),
$$

although in this case $\psi_{\alpha}$ does not have the structure of an exact eigenfunction since it involves a polynomial in both $z_{j}$ and $\bar{z}_{j}$.

Two possible choices of regularizing symmetric functions $\Phi$ mentioned in [43], giving rise to the expected [58] pairwise short-scale behavior $\sim\left|z_{j k}\right|^{\alpha}$ in $\Psi$, could be

$$
\Phi_{r_{0}}=\prod_{j<k}\left|z_{j k}\right|^{2 \alpha}\left(r_{0}^{2}+\left|z_{j k}\right|^{2}\right)^{-\alpha},
$$

with a parameter $r_{0}>0$ to be optimized over, or the parameterfree (but less smooth)

$$
\Phi_{0}=\prod_{j=1}^{N} \prod_{k=1}^{\nu-1}\left|z_{j \mathrm{nn}_{k}(j)}\right|^{\alpha},
$$

where $\mathrm{nn}_{k}(j)$ denotes the $k$ th nearest neighbor of particle $j$ [among particles of the set $A \subseteq\{1,2, \ldots, N\}$ if instead writing $\left.\mathrm{nn}_{k}(j ; A)\right]$. However, as seen below, an ansatz closer to the Bijl-Jastrow form

$$
\Phi_{\mathrm{BJ}}=\prod_{j<k} f\left(\left|z_{j k}\right|\right),
$$

or the Dyson form [60]

$$
\Phi_{\mathrm{D}}=f\left(\left|z_{21}\right|\right) f\left(\left|z_{3 \mathrm{nn}_{1}(3 ; 1,2)}\right|\right) \ldots f\left(\left|z_{N \mathrm{nn}_{1}(N ; 1,2, \ldots, N-1)}\right|\right),
$$

as used for 2D Bose gases with suitable two-particle correlations $f$ (see [64]), could be better.

We note that taking $\Phi_{0}$ as in (18) as a regulator raises the degree of the state $\Psi=\Phi_{0} \psi_{\alpha}$ for even numerators to formally [if (15) were still to hold] produce the energy

$$
\begin{aligned}
\omega(N+\operatorname{deg} \Psi) & =\left(1+\alpha(v-1)-\alpha \frac{v-1}{2}\right) \omega N \\
& =\left(1+\alpha \frac{v-1}{2}\right) \omega N,
\end{aligned}
$$

which by (11) exactly matches the lower bound (5). We also note that for odd-numerator $\alpha$ and magic (i.e., shell-filling) numbers $K$,

$$
\begin{aligned}
\omega(N+\operatorname{deg} \Psi) & \sim \omega v \frac{\sqrt{8}}{3} K^{3 / 2} \\
& =\frac{\sqrt{8}}{3} v^{-1 / 2} \omega N^{3 / 2}=\frac{\sqrt{8}}{3} \sqrt{\alpha_{*}} \omega N^{3 / 2},
\end{aligned}
$$

which matches both the average-field approximation (6) for $\alpha=\alpha_{*}$ and, for small $\alpha_{*}$ and up to the value of the numerical constant, the improved rigorous lower bound (3) that was 
recently obtained by means of a Lieb-Thirring inequality [46] as a consequence of the statistical repulsion (7).

Using the above observations, we then shift the problem of estimating the ground-state energy $E_{0}(N)$ from above for even-numerator fractions to concern only the energy of the regulator $\Phi$.

Proposition 1. Assume $\Phi \in H_{\mathrm{loc}}^{1}\left(\mathbb{R}^{2 N} ; \mathbb{R}\right)$ is such that $\Psi=$ $\Phi \psi_{\alpha} \in \mathscr{D}_{\alpha}^{N}$ and $(\nabla \Phi) \psi_{\alpha} \in L^{2}\left(\mathbb{R}^{2 N} ; \mathbb{C}^{N}\right)$, where $\alpha$ is an evennumerator fraction. Then

$$
\begin{aligned}
\left\langle\Psi, \hat{H}_{N} \Psi\right\rangle= & \int_{\mathbb{R}^{2 N}}\left(\frac{1}{2 m} \sum_{j=1}^{N}\left|D_{j} \Psi\right|^{2}+\frac{m \omega^{2}}{2}|x|^{2}|\Psi|^{2}\right) d x \\
= & \omega\left(N+\operatorname{deg} \psi_{\alpha}\right) \int_{\mathbb{R}^{2 N}}|\Psi|^{2} d x \\
& +\frac{1}{2 m} \int_{\mathbb{R}^{2 N}} \sum_{j=1}^{N}\left|\nabla_{j} \Phi\right|^{2}\left|\psi_{\alpha}\right|^{2} d x .
\end{aligned}
$$

Proof. By taking an appropriate approximating sequence in $H^{1}\left(\mathbb{R}^{2 N}\right)=H_{0}^{1}\left(\mathbb{R}^{2 N} \backslash \triangle\right)$ (see [44], Lemma 3) we may assume without loss of generality that $\Phi \in C_{c}^{\infty}\left(\mathbb{R}^{2 N} \backslash \triangle\right)$ (smooth with compact support outside $\triangle$ ). Then

$$
D_{j} \Psi=\left(-i \nabla_{j} \Phi\right) \psi_{\alpha}+\Phi D_{j} \psi_{\alpha} \in C_{c}^{\infty}\left(\mathbb{R}^{2 N} \backslash \triangle\right)
$$

and

$$
\begin{aligned}
\sum_{j} \int\left|D_{j} \Psi\right|^{2} d x= & \sum_{j} \int \overline{D_{j} \Psi} \cdot\left(-i \nabla_{j} \Phi\right) \psi_{\alpha} d x \\
& +\sum_{j} \int \overline{D_{j} \Psi} \cdot \Phi D_{j} \psi_{\alpha} d x,
\end{aligned}
$$

where, using that $\alpha \mathbf{A}_{j}$ is real and a partial integration,

$$
\begin{aligned}
\int & \overline{D_{j} \Psi} \cdot \Phi D_{j} \psi_{\alpha} d x \\
= & \int i \nabla_{j} \bar{\Psi} \cdot \Phi D_{j} \psi_{\alpha} d x+\int \alpha \mathbf{A}_{j} \bar{\Psi} \cdot \Phi D_{j} \psi_{\alpha} d x \\
= & \int \bar{\Psi}\left(-i \nabla_{j} \Phi\right) \cdot D_{j} \psi_{\alpha} d x \\
& +\int \bar{\Psi} \Phi\left(-i \nabla_{j} \cdot D_{j} \psi_{\alpha}\right) d x+\int \bar{\Psi} \Phi \alpha \mathbf{A}_{j} \cdot D_{j} \psi_{\alpha} d x \\
= & -\int \overline{\left(-i \nabla_{j} \Phi\right) \psi_{\alpha}} \cdot\left(D_{j} \Psi-\left(-i \nabla_{j} \Phi\right) \psi_{\alpha}\right) d x \\
& +\int \bar{\Psi} \Phi D_{j} \cdot D_{j} \psi_{\alpha} d x .
\end{aligned}
$$

Hence

$$
\begin{aligned}
& \int\left(\frac{1}{2 m} \sum_{j}\left|D_{j} \Psi\right|^{2}+\frac{m \omega^{2}}{2}|x|^{2}|\Psi|^{2}\right) d x \\
& =\int \bar{\Psi} \Phi \hat{H}_{N} \psi_{\alpha} d x+\frac{1}{2 m} \int \sum_{j}\left|\nabla_{j} \Phi\right|^{2}\left|\psi_{\alpha}\right|^{2} d x
\end{aligned}
$$

$$
\begin{aligned}
& +\frac{1}{2 m}\left(\int \sum_{j} \overline{D_{j} \Psi} \cdot\left(-i \nabla_{j} \Phi\right) \psi_{\alpha} d x\right. \\
& \left.-\int \sum_{j} \overline{\left(-i \nabla_{j} \Phi\right) \psi_{\alpha}} \cdot D_{j} \Psi d x\right),
\end{aligned}
$$

and by (15) it then remains to be proven that the last expression is zero. Expanding the derivative and collecting the terms, and making another partial integration [now for $\Phi^{2}=|\Phi|^{2} \in$ $\left.C_{c}^{\infty}\left(\mathbb{R}^{2 N} \backslash \triangle\right)\right]$, one finds that it equals

$$
\frac{i}{2 m} \sum_{j} \int_{\mathbb{R}^{2 N}}|\Phi|^{2} \nabla_{j} \cdot\left(\mathbf{J}_{j}\left[\psi_{\alpha}\right]+\alpha \mathbf{A}_{j}\left|\psi_{\alpha}\right|^{2}\right) d x,
$$

where $\mathbf{J}[u]:=\frac{i}{2}(u \nabla \bar{u}-\bar{u} \nabla u)$. Finally we may use that $\nabla$. $\mathbf{A}=0$ and the eigenfunction equation (15) and its complex conjugate to show that

$$
\sum_{j} \nabla_{j} \cdot\left(\mathbf{J}_{j}\left[\psi_{\alpha}\right]+\alpha \mathbf{A}_{j}\left|\psi_{\alpha}\right|^{2}\right)=0
$$

on $\mathbb{R}^{2 N} \backslash \triangle$, which proves the proposition.

The energy in the state $\Psi$ thus depends solely on the correlations of the weight $\left|\psi_{\alpha}\right|^{2}$ and its balance with the regulator $\Phi$, which is required to vanish sufficiently fast as particles come together. However, if these correlations effectively turn out to decay faster than the average interparticle spacing then, in analogy with dilute hard-core bosons [61,63], there may be room for a smaller energy (note that $\Phi$ should not have Dirichlet but rather Neumann-type boundary conditions at the interparticle scale).

\section{VI. $R$-EXTENDED ANYON GAS}

That exact eigenstates for the many-anyon problem can be found at all is far from trivial, and the reason for it to hold for the above states is that they satisfy a remarkable simplifying identity. Here we shall consider this identity in detail and greater generality, in the context of the extended anyon gas.

By an " $R$-extended anyon" we mean that we have replaced the singular Aharonov-Bohm flux on each anyon by a uniform field on a disk of finite radius $R>0$. In other words, we replace (2) by (cf. [30,45,46,52,81,82])

$$
\mathbf{A}_{j}(\mathbf{x}):=\sum_{k \neq j} \frac{\left(\mathbf{x}-\mathbf{x}_{k}\right)^{\perp}}{\left|\mathbf{x}-\mathbf{x}_{k}\right|_{R}^{2}}, \quad|\mathbf{x}|_{R}:=\max \{|\mathbf{x}|, R\},
$$

so that

$$
\operatorname{curl} \alpha \mathbf{A}_{j}(\mathbf{x})=2 \pi \alpha \sum_{k \neq j} \frac{\mathbb{1}_{D\left(\mathbf{x}_{k}, R\right)}(\mathbf{x})}{\pi R^{2}} \stackrel{R \rightarrow 0}{\longrightarrow} 2 \pi \alpha \sum_{k \neq j} \delta_{\mathbf{x}_{k}}(\mathbf{x}),
$$

where $\mathbb{1}_{D(\mathbf{y}, R)}$ denotes the indicator function on a disk of radius $R$ centered at $\mathbf{y}$. Note that this form for the magnetic interaction is actually the natural one from the perspective of emergent anyons [30], for which the size $R$ is implied by the experimental conditions. There is also a natural dimensionless parameter in the problem given by the ratio of the size of the magnetic flux to the average interparticle distance, $\bar{\gamma}:=R \bar{\varrho}^{1 / 2}$. This has been called the "magnetic filling ratio" in $[46,52,83]$. 

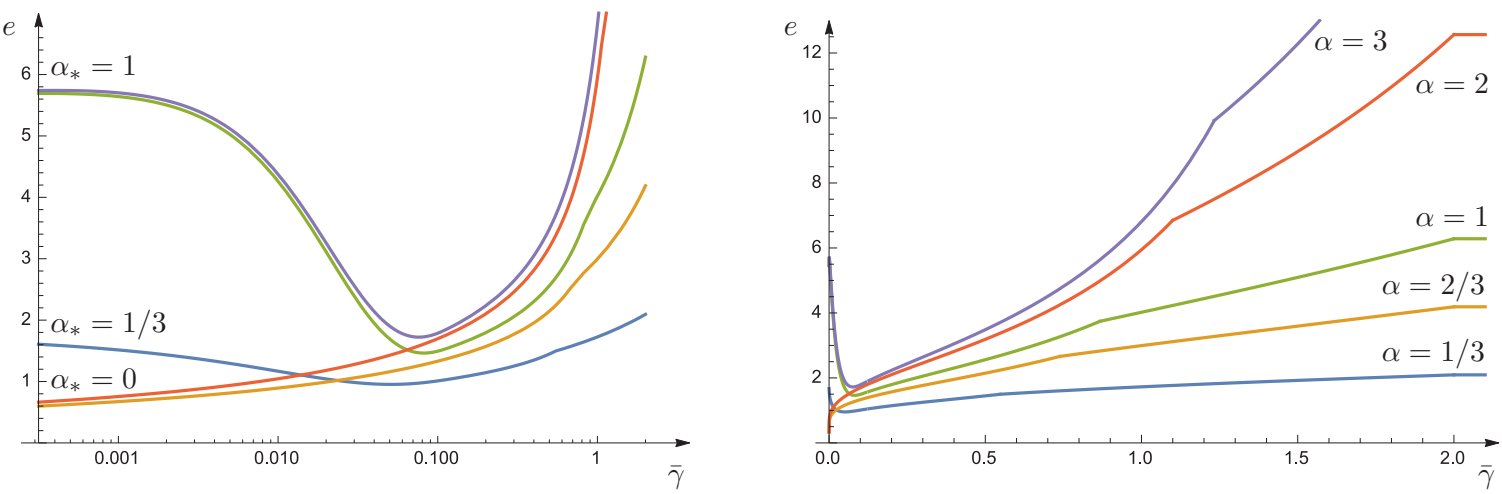

FIG. 5. Dependence on the density $\bar{\gamma}$ of the lower bound $e(\alpha, \bar{\gamma})$ for the ground-state energy of the extended anyon gas for some values of $\alpha$, with constants and scales chosen for illustrative purposes as explained in [46].

Based on the long-range local exclusion principle (7) and further short-range magnetic bounds that arise only in this extended context, it was shown rigorously in [46] that a homogeneous gas of such $R$-extended anyons satisfies in the thermodynamic limit (on a box of side length $L \rightarrow \infty$ and with fixed average density $\bar{\varrho}=N / L^{2}$ ) a universal bound for the energy per particle of the form ${ }^{4}$

$$
\liminf _{\substack{N, L \rightarrow \infty \\ N / L^{2}=\bar{\varrho}}} \frac{E_{0}(N)}{N} \geqslant C e(\alpha, \bar{\gamma}) \frac{\bar{\varrho}}{2 m}
$$

where $C$ is a positive universal constant and (dimensionless; see Fig. 5)

$e(\alpha, \bar{\gamma}) \sim \begin{cases}\frac{2 \pi}{\ln \bar{\gamma} \mid}+\pi\left(j_{\alpha_{*}}^{\prime}\right)^{2} \geqslant 2 \pi \alpha_{*}, & \bar{\gamma} \rightarrow 0(\text { fixed } \alpha \neq 0), \\ 2 \pi|\alpha|, & \bar{\gamma} \gtrsim 1 .\end{cases}$

This bound interpolates between a dilute regime where the effect of the statistical repulsion dominates (note that there is also, even for $\alpha \in 2 \mathbb{Z} \backslash\{0\}$, a strictly positive interaction energy which vanishes with the density similar to that of a hardcore 2D Bose gas), and a dense regime where the dependence on $\alpha$ matches that which is expected from average-field theory (6). In [45] it was shown that for $R$-extended anyons in an external trap $V$ and in a limit such that the filling $\bar{\gamma}$ is high but the statistics parameter $\alpha$ small ("almost-bosonic" anyons; see also [47]), the average-field approximation is a correct description in the sense that the particles become identically distributed in a self-generated magnetic field. However, given the linear dependence on the strength of the magnetic field $|\alpha|$ [our lower bound (22) is valid for any $\alpha \in \mathbb{R}$ ] for high densities, and the periodicity in $\alpha$ for ideal anyons, there must be some nontrivial interpolation between these two regimes.

Let us now introduce a convenient notation for an associated scalar (super)potential (cf. [45])

$$
w_{R}(\mathbf{x}):= \begin{cases}\ln |\mathbf{x}|, & |\mathbf{x}|>R \\ \ln R+\frac{1}{2}\left(|\mathbf{x}|^{2} / R^{2}-1\right), & |\mathbf{x}| \leqslant R\end{cases}
$$

\footnotetext{
${ }^{4}$ We are taking the lim inf (and assume Dirichlet boundary conditions) here because, although the sequence is bounded, it has not yet been proved in general that a limit exists.
}

with

$$
\nabla w_{R}(\mathbf{x})=(\mathbf{x})_{R}^{-1}:=\mathbf{x} /|\mathbf{x}|_{R}^{2}
$$

and

$$
W_{R}(\mathrm{x}):=\sum_{\substack{j, k=1 \\ j \neq k}}^{N} \Delta w_{R}\left(\mathbf{x}_{j}-\mathbf{x}_{k}\right)=2 \pi \sum_{\substack{j, k=1 \\ j \neq k}}^{N} \frac{\mathbb{1}_{D(0, R)}}{\pi R^{2}}\left(\mathbf{x}_{j}-\mathbf{x}_{k}\right) .
$$

We then have the following property, which is essentially a result concerning supersymmetry of the corresponding Pauli operator. It has been discussed in that context in [73,81]; however, we will here supply a different proof.

Proposition 2. Let $\Psi_{ \pm}(x)=e^{\mp \alpha \sum_{j<k} w_{R}\left(\mathbf{x}_{j}-\mathbf{x}_{k}\right)} f_{\mp}(z)$, where $f_{+}$is analytic ( $f_{-}$antianalytic) in all the variables $z_{j}$. Then

$$
\sum_{j=1}^{N} D_{j}^{2} \Psi_{ \pm}= \pm \alpha W_{R} \Psi_{ \pm}
$$

In particular, $\Psi_{ \pm}$are for $R=0$ generalized zero-energy eigenfunctions of the $N$-anyon kinetic energy operator $\hat{T}_{\alpha}$ considered on $\mathbb{R}^{2 N} \backslash \Delta$.

Proof. We find it convenient to work with $\mathcal{G}\left(\mathbb{C}^{2}\right)$, the complex Clifford algebra over $\mathbb{R}^{2}$, and write for the $\pi / 2$ rotation $\mathbf{x}^{\perp}=\left(x \mathbf{e}_{1}+y \mathbf{e}_{2}\right)^{\perp}=\mathbf{x} I$, i.e., multiplication from the right with the pseudoscalar $I=\mathbf{e}_{1} \mathbf{e}_{2}$. Note that

$\nabla_{j} \Psi_{ \pm}=e^{\mp \alpha \sum_{i<k} w_{R}\left(\mathbf{x}_{i}-\mathbf{x}_{k}\right)}\left(\mp \alpha \sum_{k \neq j}\left(\mathbf{x}_{j}-\mathbf{x}_{k}\right)_{R}^{-1} f_{\mp}+\nabla_{j} f_{\mp}\right)$,

and thus

$$
\begin{aligned}
D_{j} \Psi_{ \pm}= & e^{\mp \alpha \sum_{i<k} w_{R}\left(\mathbf{x}_{i}-\mathbf{x}_{k}\right)} \\
& \times\left(\alpha \sum_{k \neq j}\left(\mathbf{x}_{j}-\mathbf{x}_{k}\right)_{R}^{-1}( \pm i+I) f_{\mp}-i \nabla_{j} f_{\mp}\right) .
\end{aligned}
$$

Furthermore,

$$
\begin{aligned}
D_{j} \cdot D_{j} \Psi_{ \pm}= & {\left[\left(D_{j} e^{\mp \alpha \sum_{i<k} w_{R}\left(\mathbf{x}_{i}-\mathbf{x}_{k}\right)}\right)-i e^{\mp \alpha \sum_{i<k} w_{R}\left(\mathbf{x}_{i}-\mathbf{x}_{k}\right)} \nabla_{j}\right] } \\
& \cdot\left( \pm i \alpha \sum_{k \neq j}\left(\mathbf{x}_{j}-\mathbf{x}_{k}\right)_{R}^{-1}(1 \mp i I) f_{\mp}-i \nabla_{j} f_{\mp}\right)
\end{aligned}
$$




$$
\begin{aligned}
& = \pm \alpha \sum_{k \neq j} \nabla_{j} \cdot\left(\mathbf{x}_{j}-\mathbf{x}_{k}\right)_{R}^{-1}(1 \mp i I) \Psi_{ \pm} \\
& = \pm \alpha \sum_{k \neq j} \Delta w_{R}\left(\mathbf{x}_{j}-\mathbf{x}_{k}\right) \Psi_{ \pm},
\end{aligned}
$$

where the fundamental simplifying identity used is that for any $\mathbf{x}, \mathbf{y} \in \mathbb{C}^{2}$,

$$
\mathbf{x}(1 \pm i I) \cdot \mathbf{y}(1 \pm i I)=0
$$

since $I^{2}=-1$ and $\mathbf{x} I \cdot \mathbf{y} I=\mathbf{x} \cdot \mathbf{y}$. We have also used $\nabla$. $\nabla I=\nabla \cdot \nabla^{\perp}=0$, and that for $z_{ \pm}=x \pm i y$ and $f: \mathbb{C} \rightarrow \mathbb{C}$ analytic

$$
\nabla\left(f\left(z_{ \pm}\right)\right)=f^{\prime}\left(z_{ \pm}\right)\left(\mathbf{e}_{1} \pm i \mathbf{e}_{2}\right)=f^{\prime}\left(z_{ \pm}\right) \mathbf{e}_{1}(1 \pm i I)
$$

and $\Delta f=0$.

In the $R$-extended case we therefore take as our trial states $\Psi=\Phi \psi_{\alpha}$ with the Jastrow factor in (8) and (9) replaced by $e^{-\alpha \sum_{j<k} w_{R}\left(\mathbf{x}_{j}-\mathbf{x}_{k}\right)}$, and in the case of the homogeneous gas the $\varphi_{k}$ are taken to be the eigenstates of the Neumann Laplacian on the square $Q_{L}$ of side length $L$ (thus $\varphi_{0} \equiv L^{-1}$ ). Note that these states are regular even without the factor $\Phi$, since

$$
e^{-\alpha w_{R}(\mathbf{x})}= \begin{cases}|\mathbf{x}|^{-\alpha}, & |\mathbf{x}|>R, \\ R^{-\alpha} e^{\frac{\alpha}{2}\left(1-|\mathbf{x}|^{2} / R^{2}\right)}, & |\mathbf{x}| \leqslant R .\end{cases}
$$

However, in order to obtain the correct balance for a low total energy, and to take the appropriate limits, we expect that an additional regulator is still necessary. In particular, in the dilute limit $\bar{\gamma}=R \bar{\varrho}^{1 / 2} \rightarrow 0$ the Jastrow factor describes an attraction which needs to be turned into a short-range repulsion, as illustrated by the below reformulation of the energy in terms of $\Phi$. The proof is almost identical to that of Proposition 1, where the use of the identity (15) is replaced by Proposition 2.

Proposition 3. Assume $\Phi \in H_{0}^{1}\left(Q_{L}^{N} ; \mathbb{R}\right)$ is such that $\Psi=$ $\Phi \psi_{\alpha} \in \mathscr{D}_{\alpha}^{N}$ and $(\nabla \Phi) \psi_{\alpha} \in L^{2}\left(Q_{L}^{N}\right)$, where $\alpha \in[0,1]$ is an even-numerator fraction. Then

$$
\begin{aligned}
2 m\left\langle\Psi, \hat{T}_{\alpha} \Psi\right\rangle & =\int_{Q_{L}^{N}} \sum_{j=1}^{N}\left|D_{j} \Psi\right|^{2} d x \\
& =\int_{Q_{L}^{N}}\left(\sum_{j=1}^{N}\left|\nabla_{j} \Phi\right|^{2}+\alpha W_{R}|\Phi|^{2}\right)\left|\psi_{\alpha}\right|^{2} d x .
\end{aligned}
$$

In the dilute limit, in which the scattering length of the soft-disk potential $\alpha W_{R}$ becomes relatively small, this again seems to be able to produce a low energy for even-numerator states. Also, for odd-numerator states, naively estimating the energy of $\Psi$ in terms of that of the one-body states $\varphi_{k}$ of the Slater determinants in (9) yields the tentative bound

$$
2 m E_{0}(N) \lesssim \nu 2 \pi K^{2} / L^{2} \sim 2 \pi \alpha_{*} \bar{\varrho} N,
$$

which again matches the available lower bounds. Note also that the repulsive pair potential $\alpha W_{R}$ that emerged above matches in the dilute limit the point interaction conventionally introduced to regularize ideal anyons [84].

\section{CONCLUSIONS}

With the ansatz given by the discussed trial states, we have reduced the difficult problem of bounding the ground-state energy of a system of $N$ Abelian anyons with even-numerator rational statistics parameter to the study of the $N$ dependence of the quantity

$$
\frac{\int_{\mathbb{R}^{2 N}}\left(\sum_{j=1}^{N}\left|\nabla_{j} \Phi\right|^{2}+\alpha W_{R}|\Phi|^{2}\right)\left|\psi_{\alpha}\right|^{2} d x}{\int_{\mathbb{R}^{2 N}}|\Phi|^{2}\left|\psi_{\alpha}\right|^{2} d x},
$$

which is essentially the energy of a repulsive 2D Bose gas described by $\Phi$ but weighted by $\left|\psi_{\alpha}\right|^{2}$. One could try to estimate this using the techniques of Dingle, Jastrow, and Dyson (see [60,64] and references therein). Alternatively, Monte Carlo methods could prove useful in this formulation. In any case, since the weight $\left|\psi_{\alpha}\right|^{2}$ is designed so as to cancel any long-range correlations by means of its clustering properties, and since the energy of a dilute 2D Bose gas is logarithmically small $[61,63]$, the discussed approach indeed looks very promising. Also, if the anyons are not completely free but an additional attraction is added then it seems rather clear from the above expression with suitable $\Phi$ that they would prefer to cluster in this way.

Finally, let us remark that if these are indeed the correct (approximative) ground states for a many-body system of Abelian anyons, then they could possibly also explain from a more fundamental perspective the occurrence of such clustering states in the FQHE (cf. [15], pp. 239-240, and note that the usual Read-Rezayi states are supposed to be built of clusters of $k$ anyons with $\alpha=2 / k$ in a zero magnetic field). Furthermore, the elementary excitations of such an Abelian anyon condensed ground state may, according to well-known properties of clustering states, in turn be non-Abelian anyons.

Note added. The author has in very recent unpublished work with R. Seiringer proved that the ground-state energy of the ideal anyon gas satisfies upper and lower bounds in terms of $\alpha_{2}$, i.e., linear in $\alpha$, up to a constant which is weaker than that in previous bounds in terms of $\alpha_{*}$ such as (3) and (22). However, the arguments discussed here for the proposed trial states and the conclusions of this paper suggest that these states may actually be closer to the true ground states of the problem, and that the exact energy could be lower for even-numerator $\alpha$ than for odd numerators. Furthermore, in the scale-free ideal case the clustering behavior seems to be balanced by the energy cost of localization of the uncertainty principle, but this may well change with the addition of an attractive interaction.

\section{ACKNOWLEDGMENTS}

The idea for using the clustering states (8) and (9) for the many-anyon problem came during a postdoctoral stay at IHÉS as part of an EPDI fellowship and, upon coming across the paper in [71], I found out about their intriguing connection to the Moore-Read and Read-Rezayi states of the FQHE. I am especially grateful to Jan Philip Solovej for fruitful discussions and collaboration on this topic, and for initiating our mathematical study of anyons in the first place during my postdoc in Copenhagen. The plots of Figs. 3-5 were produced in collaboration with Simon Larson. I also thank Michele Correggi, Phan Thành Nam, Fabian Portmann, and Nicolas Rougerie for valuable discussions and collaborations on closely related subjects, as well as Eddy Ardonne, Hans 
Hansson, Thierry Jolicoeur, Stéphane Ouvry, Raoul Santachiara, Robert Seiringer, and Andrea Trombettoni for useful references, comments, and discussions. Financial support from the Knut and Alice Wallenberg Foundation, Grant No. KAW 2010.0063, and the Swedish Research Council, Grant No. 2013-4734, is gratefully acknowledged.
[1] J. M. Leinaas and J. Myrheim, On the theory of identical particles, Nuovo Cimento B 37, 1 (1977).

[2] G. A. Goldin, R. Menikoff, and D. H. Sharp, Representations of a local current algebra in nonsimply connected space and the Aharonov-Bohm effect, J. Math. Phys. 22, 1664 (1981).

[3] F. Wilczek, Magnetic Flux, Angular Momentum, and Statistics, Phys. Rev. Lett. 48, 1144 (1982).

[4] F. Wilczek, Quantum Mechanics of Fractional-Spin Particles, Phys. Rev. Lett. 49, 957 (1982).

[5] Y.-S. Wu, Multiparticle Quantum Mechanics Obeying Fractional Statistics, Phys. Rev. Lett. 53, 111 (1984).

[6] J.-M. Souriau, Structure des systèmes dynamiques, Maîtrises de mathématiques (Dunod, Paris, 1970); English translation by R. H. Cushman and G. M. Tuynman, Progress in Mathematics (Birkhäuser Boston Inc., Boston, MA, 1997), Vol. 19, http://www.jmsouriau.com/structure_des_systemes_ dynamiques.htm.

[7] G. Date, M. V. N. Murthy, and R. Vathsan, Classical and quantum mechanics of anyons, arXiv:cond-mat/0302019.

[8] S. Forte, Quantum mechanics and field theory with fractional spin and statistics, Rev. Mod. Phys. 64, 193 (1992).

[9] J. Fröhlich, Quantum Statistics and Locality, Proceedings of the Gibbs Symposium (New Haven, CT, 1989) (American Mathematical Society, Providence, RI, 1990), pp. 89-142.

[10] R. Iengo and K. Lechner, Anyon quantum mechanics and ChernSimons theory, Phys. Rep. 213, 179 (1992).

[11] A. Khare, Fractional Statistics and Quantum Theory, 2nd ed. (World Scientific, Singapore, 2005).

[12] A. Lerda, Anyons (Springer-Verlag, Berlin-Heidelberg, 1992).

[13] J. Myrheim, in Anyons, edited by A. Comtet, T. Jolicœur, S. Ouvry, and F. David, Les Houches - Ecole d'Ete de Physique Theorique (Springer-Verlag, Berlin, 1999), Vol. 69, pp. 265413.

[14] S. Ouvry, Anyons and lowest Landau level anyons, Séminaire Poincaré 11, 77 (2007).

[15] A. Stern, Anyons and the quantum Hall effect - A pedagogical review, Ann. Phys. (NY) 323, 204 (2008).

[16] F. Wilczek, Fractional Statistics and Anyon Superconductivity (World Scientific, Singapore, 1990).

[17] S. Girvin, Introduction to the fractional quantum Hall effect, Séminaire Poincaré 2, 54 (2004).

[18] M. O. Goerbig, Quantum Hall Effects, Lecture notes, 2009, arXiv:0909.1998.

[19] J. K. Jain, Composite Fermions (Cambridge University Press, Cambridge, UK, 2007).

[20] R. B. Laughlin, Nobel lecture: Fractional quantization, Rev. Mod. Phys. 71, 863 (1999).

[21] H. Störmer, D. Tsui, and A. Gossard, The fractional quantum Hall effect, Rev. Mod. Phys. 71, S298 (1999).

[22] I. Bloch, J. Dalibard, and W. Zwerger, Many-body physics with ultracold gases, Rev. Mod. Phys. 80, 885 (2008).

[23] N. R. Cooper, Rapidly rotating atomic gases, Adv. Phys. 57, 539 (2008).
[24] A. Morris and D. Feder, Gaussian Potentials Facilitate Access to Quantum Hall States in Rotating Bose Gases, Phys. Rev. Lett. 99, 240401 (2007).

[25] M. Roncaglia, M. Rizzi, and J. Dalibard, From rotating atomic rings to quantum Hall states, Sci. Rep. 1, 43 (2011).

[26] S. Viefers, Quantum Hall physics in rotating Bose-Einstein condensates, J. Phys. C 20, 123202 (2008).

[27] K. I. Bolotin, F. Ghahari, M. D. Shulman, H. L. Stormer, and P. Kim, Observation of the fractional quantum Hall effect in graphene, Nature (London) 462, 196 (2009).

[28] X. Du, I. Skachko, F. Duerr, A. Luican, and E. Y. Andrei, Fractional quantum Hall effect and insulating phase of Dirac electrons in graphene, Nature (London) 462, 192 (2009).

[29] D. Arovas, J. R. Schrieffer, and F. Wilczek, Fractional Statistics and the Quantum Hall Effect, Phys. Rev. Lett. 53, 722 (1984).

[30] D. Lundholm and N. Rougerie, Emergence of Fractional Statistics for Tracer Particles in a Laughlin Liquid, Phys. Rev. Lett. 116, 170401 (2016).

[31] N. Rougerie, Habilitation thesis, LPMMC, Université GrenobleAlpes \& CNRS, 2016, arXiv:1607.03833.

[32] D. P. Arovas, R. Schrieffer, F. Wilczek, and A. Zee, Statistical mechanics of anyons, Nucl. Phys. B 251, 117 (1985).

[33] M. Sporre, J. J. M. Verbaarschot, and I. Zahed, Numerical Solution of the Three-Anyon Problem, Phys. Rev. Lett. 67, 1813 (1991).

[34] M. V. N. Murthy, J. Law, M. Brack, and R. K. Bhaduri, Quantum Spectrum of Three Anyons in an Oscillator Potential, Phys. Rev. Lett. 67, 1817 (1991).

[35] M. Sporre, J. J. M. Verbaarschot, and I. Zahed, Four anyons in a harmonic well, Phys. Rev. B 46, 5738 (1992).

[36] M. Sporre, J. Verbaarschot, and I. Zahed, Anyon spectra and the third virial coefficient, Nucl. Phys. B 389, 645 (1993).

[37] Y. H. Chen, F. Wilczek, E. Witten, and B. I. Halperin, On anyon superconductivity, Int. J. Mod. Phys. B 03, 1001 (1989).

[38] Y. Hosotani, Neutral and charged anyon fluids, Int. J. Mod. Phys. B 07, 2219 (1993).

[39] X. G. Wen and A. Zee, Compressibility and superfluidity in the fractional-statistics liquid, Phys. Rev. B 41, 240 (1990).

[40] A. Dasnières de Veigy and S. Ouvry, Equation of State of an Anyon Gas in a Strong Magnetic Field, Phys. Rev. Lett. 72, 600 (1994).

[41] W. R. Minor, Ground-state energy of a dilute anyon gas, Phys. Rev. B 47, 12716 (1993).

[42] D. Lundholm and J. P. Solovej, Hardy and Lieb-Thirring inequalities for anyons, Commun. Math. Phys. 322, 883 (2013).

[43] D. Lundholm and J. P. Solovej, Local exclusion principle for identical particles obeying intermediate and fractional statistics, Phys. Rev. A 88, 062106 (2013).

[44] D. Lundholm and J. P. Solovej, Local exclusion and LiebThirring inequalities for intermediate and fractional statistics, Ann. Henri Poincaré 15, 1061 (2014).

[45] D. Lundholm and N. Rougerie, The average field approximation for almost bosonic extended anyons, J. Stat. Phys. 161, 1236 (2015). 
[46] S. Larson and D. Lundholm, Exclusion bounds for extended anyons, arXiv:1608.04684.

[47] M. Correggi, D. Lundholm, and N. Rougerie, Local density approximation for the almost-bosonic anyon gas, Anal. PDE (to be published), arXiv:1611.00942.

[48] G. Gentile, Osservazioni sopra le statistiche intermedie, Nuovo Cimento 17, 493 (1940).

[49] G. Gentile, Le statistiche intermedie e le proprietà dell'elio liquido, Nuovo Cimento 19, 109 (1942).

[50] R. Chitra and D. Sen, Ground state of many anyons in a harmonic potential, Phys. Rev. B 46, 10923 (1992).

[51] D. Sen, Some supersymmetric features in the spectrum of anyons in a harmonic potential, Phys. Rev. D 46, 1846 (1992).

[52] C. Trugenberger, Ground state and collective excitations of extended anyons, Phys. Lett. B 288, 121 (1992).

[53] F. D. M. Haldane, "Fractional Statistics" in Arbitrary Dimensions: A Generalization of the Pauli Principle, Phys. Rev. Lett. 67, 937 (1991).

[54] S. B. Isakov, Statistical Mechanics for a Class of Quantum Statistics, Phys. Rev. Lett. 73, 2150 (1994).

[55] Y.-S. Wu, Statistical Distribution for Generalized Ideal Gas of Fractional-Statistics Particles, Phys. Rev. Lett. 73, 922 (1994).

[56] T. H. Hansson, J. M. Leinaas, and S. Viefers, Exclusion Statistics in a Trapped Two-Dimensional Bose Gas, Phys. Rev. Lett. 86, 2930 (2001).

[57] G. S. Canright and M. D. Johnson, Fractional statistics: alpha to beta, J. Phys. A: Math. Gen. 27, 3579 (1994).

[58] M. Bourdeau and R. D. Sorkin, When can identical particles collide?, Phys. Rev. D 45, 687 (1992).

[59] T. D. Lee and C. N. Yang, Many-body problem in quantum mechanics and quantum statistical mechanics, Phys. Rev. 105, 1119 (1957).

[60] F. J. Dyson, Ground-state energy of a hard-sphere gas, Phys. Rev. 106, 20 (1957).

[61] M. Schick, Two-dimensional system of hard-core bosons, Phys. Rev. A 3, 1067 (1971).

[62] E. H. Lieb and J. Yngvason, Ground State Energy of the Low Density Bose Gas, Phys. Rev. Lett. 80, 2504 (1998).

[63] E. H. Lieb and J. Yngvason, The ground state energy of a dilute two-dimensional Bose gas, J. Stat. Phys. 103, 509 (2001), special issue dedicated to the memory of Joaquin M. Luttinger.

[64] E. H. Lieb, R. Seiringer, J. P. Solovej, and J. Yngvason, The mathematics of the Bose gas and its condensation, Oberwolfach Seminars, Birkhäuser, 2005, arXiv:cond-mat/0610117.

[65] G. 't Hooft, Non-perturbative 2 particle scattering amplitudes in 2+1 dimensional quantum gravity, Commun. Math. Phys. 117, 685 (1988).
[66] S. Deser and R. Jackiw, Classical and quantum scattering on a cone, Commun. Math. Phys. 118, 495 (1988).

[67] B. S. Kay and U. M. Studer, Boundary conditions for quantum mechanics on cones and fields around cosmic strings, Commun. Math. Phys. 139, 103 (1991).

[68] E. H. Lieb and W. E. Thirring, Bound for the Kinetic Energy of Fermions Which Proves the Stability of Matter, Phys. Rev. Lett. 35, 687 (1975).

[69] N. Regnault, M. O. Goerbig, and T. Jolicoeur, Bridge Between Abelian and Non-Abelian Fractional Quantum Hall States, Phys. Rev. Lett. 101, 066803 (2008).

[70] N. Read and E. Rezayi, Beyond paired quantum Hall states: Parafermions and incompressible states in the first excited Landau level, Phys. Rev. B 59, 8084 (1999).

[71] A. Cappelli, L. S. Georgiev, and I. T. Todorov, Parafermion Hall states from coset projections of Abelian conformal theories, Nucl. Phys. B 599, 499 (2001).

[72] G. Moore and N. Read, Non-Abelions in the fractional quantum Hall effect, Nucl. Phys. B 360, 362 (1991).

[73] S. M. Girvin, A. H. MacDonald, M. P. A. Fisher, S.-J. Rey, and J. P. Sethna, Exactly Soluble Model of Fractional Statistics, Phys. Rev. Lett. 65, 1671 (1990).

[74] T. H. Hansson, M. Hermanns, S. H. Simon, and S. F. Viefers, Quantum Hall physics: Hierarchies and conformal field theory techniques, Rev. Mod. Phys. 89, 025005 (2017).

[75] E. Ardonne, R. Kedem, and M. Stone, Filling the Bose sea: symmetric quantum Hall edge states and affine characters, J. Phys. A: Math. Gen. 38, 617 (2005).

[76] B. A. Bernevig and F. D. M. Haldane, Model Fractional Quantum Hall States and Jack Polynomials, Phys. Rev. Lett. 100, 246802 (2008).

[77] D. Lundholm, Anyon wave functions and probability distributions, Report No. IHES/P/13/25, http://preprints.ihes.fr/2013/ P/P-13-25.pdf.

[78] C. Chou, Multi-anyon quantum mechanics and fractional statistics, Phys. Lett. A 155, 245 (1991).

[79] C. Chou, Multianyon spectra and wave functions, Phys. Rev. D 44, 2533 (1991).

[80] M. V. N. Murthy, J. Law, R. K. Bhaduri, and G. Date, On a class of noninterpolating solutions of the many-anyon problem, J. Phys. A: Math. Gen. 25, 6163 (1992).

[81] M. Y. Choi, C. Lee, and J. Lee, Soluble many-body systems with flux-tube interactions in an arbitrary external magnetic field, Phys. Rev. B 46, 1489 (1992).

[82] S. Mashkevich, Finite-size anyons and perturbation theory, Phys. Rev. D 54, 6537 (1996).

[83] C. Trugenberger, The anyon fluid in the Bogoliubov approximation, Phys. Rev. D 45, 3807 (1992).

[84] S. Ouvry, $\delta$-perturbative interactions in the Aharonov-Bohm and anyons models, Phys. Rev. D 50, 5296 (1994). 